\title{
Gene discovery, evolutionary affinity and molecular detection of Oxyspirura petrowi, an eye worm parasite of game birds
}

\author{
Lixin Xiang ${ }^{1,2^{*}}$, Fengguang Guo ${ }^{1}$, Haili Zhang ${ }^{1}$, Lloyd LaCoste ${ }^{3}$, Dale Rollins ${ }^{3,4}$, Andrea Bruno ${ }^{5}$,
} Alan M Fedynich ${ }^{5}$ and Guan Zhu ${ }^{1 *}$

\begin{abstract}
Background: Oxyspirura petrowi appears to be emerging as a nematode parasite that could negatively impact Northern Bobwhite quail individuals and populations within Texas and other regions of the United States. Despite this eye worm's potential importance in the conservation of wild quail, little is known about the general biology and genome composition of $O$. petrowi. To fill the knowledge gap, we performed a small scale random genome sequence survey, sequenced its $18 \mathrm{~S}$ rRNA and the intergenic region between the $18 \mathrm{~S}$ and $28 \mathrm{~S}$ rRNA genes, studied its phylogenetic affinity, and developed a PCR protocol for the detection of this eye worm.

Results: We have generated $\sim 240 \mathrm{~kb}$ of genome sequence data derived from 348 clones by a random genome survey of an O. petrowi genomic library. The eye worm genome is AT-rich (i.e., 62.2\% AT-content), and contains a high number of microsatellite sequences. The discovered genes encode a wide-range of proteins including hypothetical proteins, enzymes, nematode-specific proteins. Phylogenetic analysis based on 18S rRNA sequences indicate that the Spiruroidea is paraphyletic, in which Oxyspirura and its closely related species are sisters to the filarial nematodes. We have also developed a PCR protocol based on the ITS2 sequence that allows sensitive and specific detection of eye worm DNA in feces. Using this newly developed protocol, we have determined that 28\% to 33\% of the fecal samples collected from Northern Bobwhites and Scaled Quail in Texas in the spring of 2013 are O. petrowi positive.
\end{abstract}

Conclusions: The O. petrowi genome is rich in microsatellite sequences that may be used in future genotyping and molecular fingerprinting analysis. This eye worm is evolutionarily close to the filarial nematodes, implying that therapeutic strategies for filariasis such as Loa loa would be referential in developing treatments for the Thelazoidea parasites. Our qPCR-based survey has confirmed that $O$. petrowi infection is of potential concern to quail managers in Texas.

\section{Background}

Oxyspirura petrowi is a spirurian nematode (Order Spirurida) that infects the eyes of quail and other birds [1]. In Texas, a $47-56 \%$ prevalence has been reported in Northern Bobwhites (Colinus virginianus) and Scaled Quail (Callipepla squamata) [2-4]. Similar infections caused by this genus of parasites have also been reported in other animals including poultry and zoo animals, where

\footnotetext{
*Correspondence: xianglx@zju.edu.cn; gzhu@cvm.tamu.edu

'Department of Veterinary Pathobiology, College of Veterinary Medicine \& Biomedical Sciences, Texas A\&M University, College Station, Texas, USA ${ }^{2}$ College of Life Sciences, Zhejiang University, Hangzhou, Zhejiang, China Full list of author information is available at the end of the article
}

some of them were described as ocular oxyspiruriasis or oxyspirurosis [5-10]. Given that bobwhites are experiencing long-term declines throughout their range in North America, there is a recognition that populations are declining even where suitable habitat conditions exist (e.g., Rolling Plains ecoregion of Texas), thereby raising concerns that parasites such as $O$. petrowi may be a contributing factor (e.g., see a more detailed description at http:// www.quailresearch.org). It is likely that infection may cause host eye damage and physically impair vision, making birds less competitive in feeding and more susceptible to predators (Figure 1).

Although the eye worm has been considered as a possible contributing factor for the decline of wild quail

\section{Biomed Central}




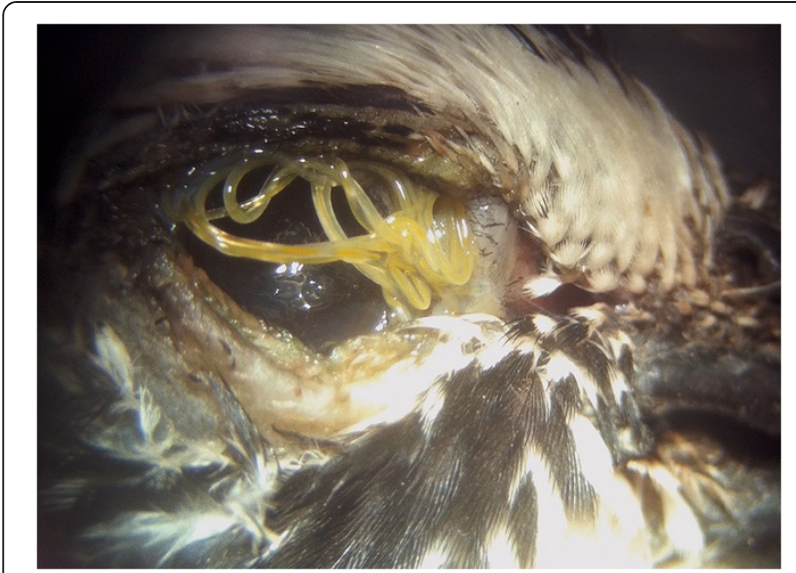

Figure 1 Oxyspirura petrowi adult worms in the eye of a Northern Bobwhite collected in Texas in February, 2013 demonstrating their potential to cause visual obstruction in addition to a pathological response resulting from infection.

populations in the Rolling Plains, little is known of the parasite's biology, particularly at the molecular and genomic levels (i.e., no molecular data were available in the GenBank databases prior to this study). Previous knowledge on the relationship of this parasite with other nematodes was solely acquired by morphology, which also needs to be validated at the molecular level. In fact, only a single nucleotide sequence is present in the database for the whole genus Oxyspirura (i.e., a 689-bp partial rRNA gene from O. conjuctivalis [GenBank: EF417873]). The lack of molecular data severely hampers our efforts in studying molecular epidemiology and transmission routes of $O$. petrowi, which may be useful for developing effective strategies to treat and control ocular oxyspiruriasis in wild quail.

To fill the knowledge gap, we have performed a smallscale genome sequence survey (GSS) that provides the first batch of genomic sequence data for this nematode. Additionally, we have cloned the $18 \mathrm{~S}$ rRNA, internal transcribed spacer 1 (ITS1), 5.8S rRNA, ITS2 and partial $28 \mathrm{~S}$ rRNA genes. The small random GSS effort rapidly generated $\sim 240 \mathrm{~kb}$ of sequence information that provided not only a snapshot of the quail eye worm genome, but also a large amount of microsatellite sequences for future genotyping and population genetic analysis. Based on the newly obtained rRNA sequences, we determined the evolutionary affinity of Oxyspirura with other nematodes among spirurians with available $18 \mathrm{~S}$ rRNA sequences. We also designed specific primers based on the ITS2 sequences, and performed real-time quantitative PCR (qPCR)-based molecular detection of O. petrowi from DNA extracted from fecal samples collected from Northern Bobwhite and Scaled Quail in Texas.

Understanding the biology of this parasitic nematode at molecular levels will enable us to effectively determine the prevalence by detecting parasite-specific DNA in feces, as well as to identify infected intermediate hosts that is otherwise difficult (if not impossible) based on morphology of larval stages. Molecular tools would enable further study of potential drug targets and targetbased drug discovery to treat this important nematode.

\section{Methods}

\section{Isolation of genomic DNA and genome sequence survey}

Adult O. petrowi worms were isolated from the eyes of Northern Bobwhites collected in Texas as part of a 3-year integrated research project called Operation Idiopathic Decline, which was initiated to further our understanding of potentially pathogenic parasites occurring within the Rolling Plains Ecoregion of Texas and western Oklahoma. All animal experiments were performed in accordance with procedures approved by the Institutional Animal Care and Use Committee of Texas A\&M University (protocol \# 2011-193).

After microscopic examination for species validation, four worms were rinsed with PBS, placed in lysis buffer of the DNeasy Blood \& Tissue Kit (Qiagen Inc., Valencia, $\mathrm{CA})$, and grinded with a plastic microtube grinder. Genomic DNA (gDNA) was isolated from the ground worms according to manufacturer's protocol for animal tissues. For the construction of a genomic library, gDNA was first subjected to whole genome amplification using a GenomePlex Complete Whole Genome Amplification (WGA) kit according the manufacturer's standard protocol (Sigma-Aldrich Co., St. Louis, MO). Amplified gDNA products were fractionated in agarose gels and fractions containing fragments between $0.5-2 \mathrm{~kb}$ were collected and purified using a Gel Extraction Kit (Omega Bio-Tek, Norcross, GA). After an incubation at $72^{\circ} \mathrm{C}$ for $20 \mathrm{~min}$ in a regular PCR reaction buffer to add a single adenine overhang to the 3'-end, the products were ligated into PCR2.1TOPO vector using a TOPO-TA cloning kit (Invitrogen, Life Technologies, Grand Island, NY).

After transformation, Escherichia coli OneShot TOP10F' chemically competent cells (Invitrogen) were plated onto LB plates spread with $40 \mu \mathrm{L}$ of $40 \mathrm{mg} / \mathrm{mL} \mathrm{X-gal} \mathrm{and} 5 \mu \mathrm{L}$ of $200 \mathrm{mM} / \mathrm{mL}$ IPTG, and incubated at $37^{\circ} \mathrm{C}$ overnight. Bacteria from a single white colony were collected into $10 \mu \mathrm{L}$ Milli-Q water in a microtube, from which $2 \mu \mathrm{L}$ of suspension was used directly as template in PCR reactions to determine the presence of inserts using a pair of M13 forward and M13 reverse primers. Colonies containing inserts with desired sizes were further incubated in LB broth containing $50 \mu \mathrm{g} / \mathrm{mL}$ kanamycin. Plasmids were prepared using a Plasmid DNA Mini kit (Omega Bio-Tek), and sequenced by the Sanger dye-terminator sequencing method at the Gene Technology Laboratory at Texas A\&M University.

Vector and adaptor sequences were removed using a cross-match algorithm, and long inserts were assembled 
using the Phrap method implemented in the MacVector program (version 12.7.4) (http://www.macvector.com). All sequences were used as queries to search the nonredundant protein and nucleotide databases at the National Center for Biotechnology Information (NCBI) by the BLA STN, BLASTX and TBLASTX algorithms using the Kori Blast program (version 3.4) (http://www.korilog.com). Additional annotations were performed using the Blast2GO program (http://www.blast2go.com/b2ghome), which included InterProScan for identifying protein domains and gene ontology (GO) analysis. GO_slim was performed at the CateGOrizer server (http://www.animalgenome.org/ cgi-bin/util/gotreei) [11]. Contigs were also mapped onto the metabolic pathways at the Kyoto Encyclopedia of Genes and Genomes (KEGG) using the KEGG Automatic Annotation Server (KAAS) (http://www.genome.jp/tools/kaas/) [12]. Candidate tRNA sequences were examined at the tRNAscan-SE server (http://lowelab.ucsc.edu/tRNAscan$\mathrm{SE} /$ ) [13]. Microsatellite sequences (also known as simple sequence repeats, SSRs) were identified using the Phobos (version 3.3.12) program (http://www.ruhr-uni-bochum.de/ spezzoo/cm/cm_phobos.htm), in which only perfect matches with a minimal length of $8 \mathrm{nt}$ and a minimal score at 8 were reported.

\section{Molecular cloning of parasite ribosomal RNA (rRNA) genes}

The 18S rRNA gene and downstream ITS1, 5.8S rRNA and ITS2 regions from $O$. petrowi were cloned by PCR using two pairs of primers: 1) nema18S_F01 (5'-CCA TGC AWG TCT AWG TTC AAA-3') and nema18S_R01 (5'-GGA AAC CTT GTT ACG ACT TTT G-3') for the nearly whole 18 S region; and 2) nema18S_F1400 (5'-GTC TGT GAT GCC CTT AGA TG-3') and nema28S_R68 (5'-TTA GTT TCT TTT CCT CCG CTT A-3') for the region between the $18 \mathrm{~S}$ and $28 \mathrm{~S}$ rRNA genes. PCR was performed using a JumpStart REDTaq ReadyMix PCR Reaction kit containing hot-start high-fidelity DNA polymerase (Sigma-Aldrich). After treating with regular Taq DNA polymerase at $72^{\circ} \mathrm{C}$ for $10 \mathrm{~min}, \mathrm{PCR}$ amplicons were similarly cloned into the pCR2.1-TOPO vector as described above. At least 10 independent clones from each reaction were sequenced, and all reads were assembled by Phrap as described above. Regions representing 18S, ITS1, $5.8 \mathrm{~S}$, ITS2 and partial $28 \mathrm{~S}$ sequences were determined by Rfam (http://rfam.janelia.org) [14].

\section{Phylogenetic reconstructions}

The assembled O. petrowi $18 \mathrm{~S}$ rRNA sequence was used as a query to search and identity nematode orthologs from the NCBI nucleotide databases. Up to 1,000 gene sequences were initially retrieved, subjected to multiple sequence alignments using the MUSCLE program (version 3.8.31) (http://drive5.com/muscle/) [15,16], followed by simple phylogenetic reconstructions by the neighborjoining (NJ) method using the Tamura-Nei nucleotide substitution model. Based on the alignment and NJ trees, short or identical sequences were individually removed, and the same procedure was repeated until a balanced dataset containing 111 sequences representing all major nematode taxonomic groups were identified. The dataset was subjected to phylogenetic reconstructions by Bayesian inference (BI) using MrBayes (version 3.2) (http://mrbayes.sourceforge.net) and the maximum likelihood (ML) method using TreeFinder (version 2008) (http://www.treefinder.de) $[17,18]$. This approach determined the phylogenetic relationships among major taxonomic groups, in which $O$. petrowi was placed within the spirurians, but the relationship among spirurians was not well resolved. Therefore, we resampled the sequences to include only taxa within Spirurida and Ascaridida as these two groups displayed a sister relationship by this study and previous analyses $[19,20]$. This also allowed us to include more taxa within these two groups. The second dataset contained 112 taxa with 1,544 nucleotide positions and was subjected to phylogenetic reconstructions using BI and ML methods. To further resolve the O. petrowi position, we also compiled a third dataset containing only taxa with close relationship with $O$. petrowi. This small dataset included only 35 taxa with 1,599 nucleotide positions, and was also subjected to $\mathrm{BI}$ and ML analyses.

In all datasets, gaps were removed and only positions that could be unambiguously aligned were used in subsequent phylogenetic analyses. In the BI analysis, 1.5 million generations of searches for the first and second datasets (or 1.0 million generations for the smaller third dataset) were performed with 4 independent chains running. Searches reached convergence as determined by the average standard deviation (SD) of split frequencies reaching $<0.01$, and potential scale reduction factor (PSRF) values for various approaching 1.0 [21]. Bootstrapping ML analyses were derived from 200 replicated sequences. In both $\mathrm{BI}$ and ML methods, the general time reversal (GTR) nucleotide substitution model was used with the consideration of fraction of invariance and 4-rate of discrete gamma (i.e., $\mathbf{G T R}+\boldsymbol{F}_{\mathrm{inv}}+\boldsymbol{\Gamma}_{\mathbf{4}}$ ). Majority rule consensus trees were visualized using FigTree (version 1.4), followed by tree annotations using Adobe Illustrator CS4.

\section{Molecular detection of $O$. petrowi}

Sequence comparison of the rRNA regions between $O$. petrowi and other nematodes indicated that 18S rRNA sequences were less suitable for designing species-specific primers, as they were highly conserved among nematodes. We hence designed primers based on the ITS2 region sequences for specific molecular detection for O. petrowi: QEW_2417F (5'-GGA TTT GCA AGA ATT GTT TCC-3') 
and QEW_2578R (5'-AAC GTT ATT GTT GCC ATA TGC-3') with a predicted product size of $162 \mathrm{bp}$. To ensure desired specificity of detection, we used DNA samples isolated from Aulonocephalus pennula, a cecal worm collected from bobwhite quail in northern Texas as negative control.

We applied real-time quantitative PCR (qPCR) to detect eye worm DNA from fecal samples from Northern Bobwhite and Scaled Quail in Texas. Feces from individual or pooled birds were collected at Rolling Plains Quail Research Ranch (RPQRR) in Fisher County, Texas in the Spring, 2013 via the seasonal trap-and-release program in a separate conservation research project. Feces were mixed, weighed and placed in lysis buffer included in the QIAamp DNA Stool Mini Kit (Qiagen). After one freeze/thaw cycle in liquid nitrogen, samples were homogenized with glass beads in a Mini-Beadbeater-16 (BioSpec Products, Inc., Bartlesville, OK) at full-speed for $4 \mathrm{~min}$, followed by DNA isolation according to the manufacturer's protocol for the stool DNA isolation kit. A SYBR-green-based qPCR detection was performed in $20 \mu \mathrm{L}$ reactions containing iQ SYBR Green Supermix reagent (Bio-Rad, Hercules, CA), the QEW_2417F/ QEW_2578R primer pair (each at $100 \mathrm{nM}$ ) and $2 \mu \mathrm{L}$ stool DNA in a Bio-Rad iCycle iQ Real-Time PCR Detection System. The reactions started with sample denaturation at $95^{\circ} \mathrm{C}$ for $5 \mathrm{~min}$, followed by 45 thermal cycles at $95^{\circ} \mathrm{C}$ for $30 \mathrm{sec}, 56^{\circ} \mathrm{C}$ for $30 \mathrm{sec}$, and $72^{\circ} \mathrm{C}$ for $30 \mathrm{sec}$. Specificity of detection was confirmed by melting curve analysis and agarose gel electrophoresis of selected PCR products. Selected individual or pooled PCR products were also sequenced to confirm their identities.

\section{Nucleotide sequence accession numbers}

Nucleotide sequences generated in this study were deposited into the GenBank database with accession numbers [GenBank:KF110799] and [GenBank:KF110800] for rRNA sequences containing type 1 and type 2 ITS1, and [GenBank:KG007611] to [GenBank:KG007945] for GSS sequences.

\section{Results and discussion}

\section{Characterization of the $O$. petrowi genome}

In this small genome sequence survey, we have obtained valid sequences for 354 clones. Among them, six sequences were determined to be bacterial contaminants, representing $1.6 \%$ of the sequenced clones. There were no contaminants from birds and other organisms, suggesting that the prepared $O$. petrowi genomic libraries were of high quality. The limited bacterial sequences (top hits were mainly Ralstonia and Caulobacter vibrioides) were likely derived from commensal bacteria in O. petrowi.

The remaining 348 valid $O$. petrowi sequences resulted in 237,239 bp of genomic sequences, which ranged from 81 to $1,220 \mathrm{bp}$ (median length $=706 \mathrm{bp}$ ) for individual contigs. The scale of the survey was relatively small, but it was sufficient to provide a first snapshot of the genome features for this nematode. The $O$. petrowi genome was generally AT-rich with GC contents ranging from $18 \%$ to $64 \%$ for individual contigs (overall mean of GC content $=37.8 \%$, vs. $56 \%$ to $70 \%$ for the six bacterial contaminants). Various BLAST searches yielded no hits for 137 contigs ( 39\%), suggesting that these sequences might be unique to Oxyspirura or closely related species. Among the 211 contigs with hits from the databases, 193 (91.5\%) had top hits on nematode sequences, particularly on those from Loa, Brugia and Wuchereria, but only very few on Ascaris or Caenorhabditis, which was congruent with the evolutionary relationship of Oxyspirura with filarioidea, Ascaridida and Rhabditida (see Additional file 1: Table S1 for a complete list of all contigs with annotations and corresponding BLAST top hits).

By combining BLAST with InterProScan searches, more than half of the contigs with hits were able to be assigned into major functional categories (i.e., 121 out of 211 contigs) (Figure 2). The functionally undefined 90 contigs were mainly hypothetical proteins with some containing low complexity sequences. Among the 121 annotatable contigs, the largest group was enzymes (total 40) that were separated into general enzymes involved in various metabolic pathways $(\mathrm{n}=30)$ and those involved in protein metabolism such as protein kinases $(\mathrm{n}=8)$ and proteases $(n=2)$. Examples of enzymes included glycogen synthase (contig QEW_195), glycosyl-transferase (QEW_224), histone acetyltranferase (QEW_156), and succinate dehydrogenase (QEW_315); while those for protein metabolism included a Ulp1 protease family member (QEW_129), as well as protein kinases in the AGC/NDR (QEW_74), CAM/CAMKL/NUAK (QEW_372) and CK1/WORM6 (QEW_249) families. Fourteen contigs encoded proteins involved in DNA/RNA metabolism, e.g., splicing factor 1 (QEW_306), DNA replication licensing factor $\mathrm{mcm}-6$ (QEW_340), and protein containing double-stranded RNA binding motif (QEW_379). There were 12 contigs containing genes encoding extracellular membrane (ECM) proteins, in which 9 contigs were associated with the nematode-specific cuticle formation, such as cuticle collagen precursors (QEW_58, QEW_59 and QEW_135), cuticulin-1 (QEW_104), and nematode cuticle collage domain containing proteins (e.g., QEW_80 and QEW_386). Other groups include those involved in ribosomal biogenesis $(n=11)$, molecular interactions $(n=8)$, ion transporters $(n=6)$, cytoskeletal proteins $(n=6)$, membrane proteins associated with cell adhesion $(\mathrm{n}=3)$, and those involved in gene expression $(n=2)$.

Interestingly, there were three contigs encoding nematode-specific major sperm protein (MSP), which was 


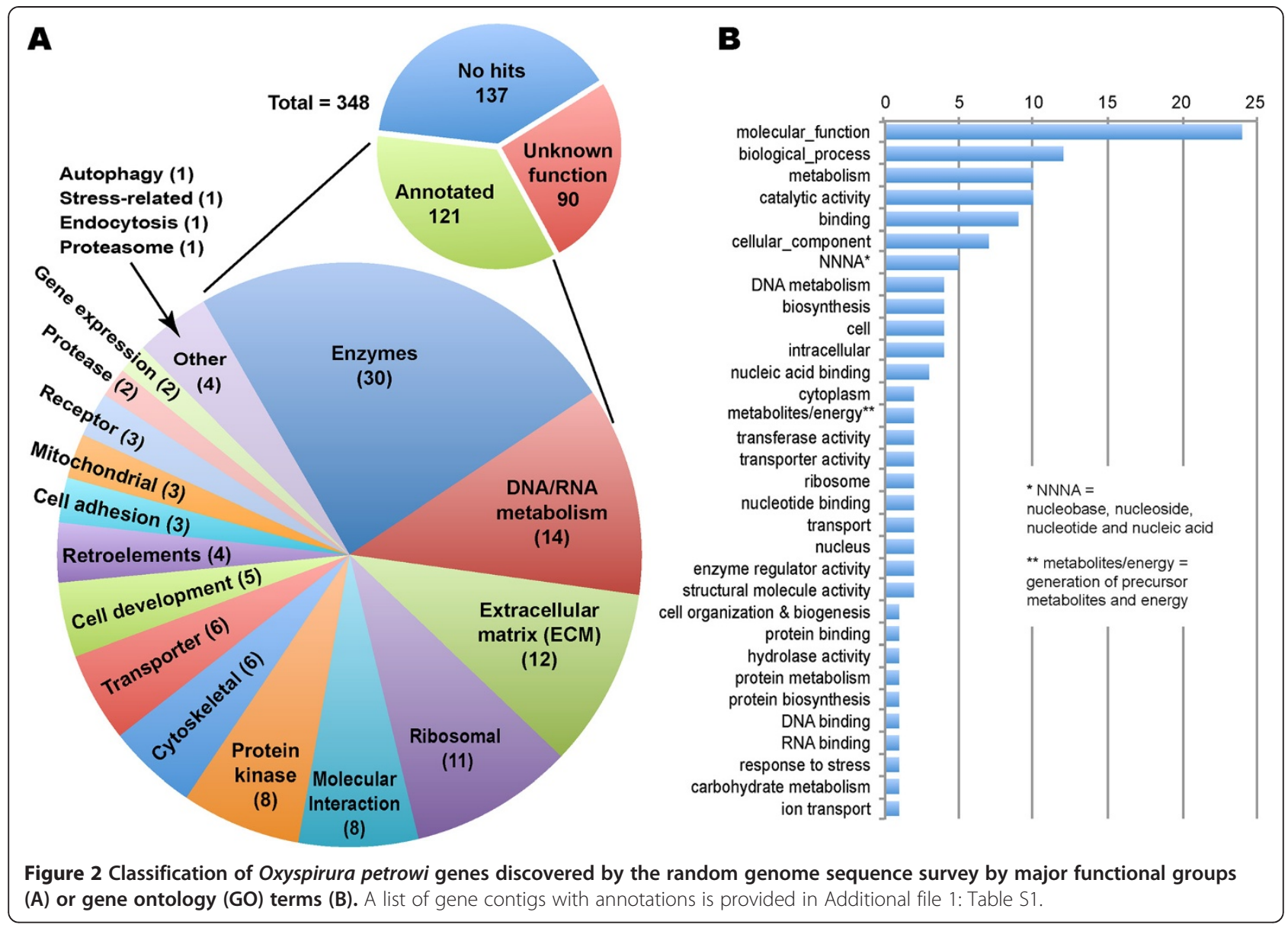

grouped together with a fic protein under the "cell development" category in Figure 2. There were also three contigs derived from the mitochondrial genomes, including gene fragments encoding cytochrome b, cytochrome $\mathrm{c}$ oxidase subunit IV (COX-IV) and NAHD dehydrogenase subunit 5 . Finally, 4 contigs were found to contain retroelements, such as tigger transposable element-derived protein 1-like proteins (QEW_112 and QEW_119) and retrotransposon protein (QEW_172).

Microsatellite sequences identified by the genome survey One major purpose of the study was to identify microsatellite sequences (also known as simple sequence repeats, SSRs) for future genotyping and population genetics analyses on this quail eye worm. In fact, $O$. petrowi appears to be rich in microsatellites, in which a total of 335 units of perfect SSRs were identified with a minimal length of 8 nt (Table 1). These included mononucleotides (228 units), dinucleotides (30), trinucleotides (56), tetranucleotides (11) and 10 repeats with 5-8 nucleotides. At least 98 contigs contained two or more SSRs, and 31 contigs contained 3-6 SSRs (Table 1). Examples included QEW_123 with 5 for mono-, tri- or tetranucleotide SSRs; QEW_126 with 5 mono-, tetra- or octa-nucleotide SSRs, and QEW_203 with 6 di-, tri- or penta-nucleotide SSRs (see Additional file 2: Table S2 for a complete list of detected microsatellite sequences). We also looked at the distribution of microsatellites with repeat units of $\geq 2 \mathrm{nt}$, which revealed $\sim 2$ or $\sim 1.5$ times more microsatellite sequences are present in contigs with no hits in BLAST/InterProScan searches (19.0\%) or with hits but unknown function (14.4\%) than in the

Table 1 Statistics on the lengths of repeat units and numbers of microsatellite sequences per contig in Oxyspirura petrowi identified by the genome sequence survey

\begin{tabular}{cccc}
\hline $\begin{array}{c}\text { Length of } \\
\text { repeat unit }\end{array}$ & Counts & $\begin{array}{c}\text { No. microsatellites } \\
\text { per contig }\end{array}$ & Counts \\
\hline 1 & 228 & 1 & 86 \\
2 & 30 & 2 & 67 \\
3 & 56 & 3 & 17 \\
4 & 11 & 4 & 7 \\
5 & 2 & 5 & 6 \\
6 & 6 & 6 & 1 \\
8 & 2 & $\geq 7$ & 0 \\
Total microsatellites & 335 & Average no. per contig & $\mathbf{1 . 8 2}$ \\
\hline
\end{tabular}


annotatable contigs (9.9\%) (Table 2). In summary, the eye worm genome contains a rich number of microsatellite sequences with the potential to be further validated as potential genetic markers.

\section{Phylogenetic position of 0 . petrowi based on $18 \mathrm{~S}$ rRNA genes}

Our first phylogenetic analyses based on a large $18 \mathrm{~S}$ rRNA dataset with BI and ML methods produced trees that agreed with those produced by others. While $O$. petrowi was clustered within the Spirurida clade, it was close to a branch consisting of Tetrameres fissipina and an unknown Onchoceridae species. This was likely a result caused by a long branch attraction (LBA) artifact based on the unusual long branch formed by $T$. fissipina and the Onchoceridae species, as well as by the obvious high numbers of nucleotide substitutions in these two sequences (data not shown). We also observed potential sequencing mistakes for the long $18 \mathrm{~S}$ rRNA sequence of Thelazia lacrymalis (DQ503458). Therefore, we removed these three sequences from subsequent analyses. Because Spirurida was consistently placed as a sister group with Ascaridida, as observed in this study and by others $[19,20]$, our second dataset contained only sequences from these two groups, which allowed more reliable alignment with more nucleotide positions to better resolve the position of O. petrowi within Spirurida using Ascaridida as outgroup. Gnathastoma sequences were also excluded from the second dataset, as they have been shown to be seperate from the rest of the spirurids $[19,20]$.

Both $\mathrm{BI}$ and $\mathrm{ML}$ trees inferred from the second dataset distinctly separated Ascaridida from Spirurida (Figure 3A). Within the Spirurida clade, Dracunculoidea and Camallanoidea formed two major sister branches, whereas the third branch comprised of the remaining families including Spiruroidea, Acuarioidea, Physalopteroidea, Filarioidea, Habronematoidea and Thelazioidea. Further phylogenetic analysis based only on sequences from the third branch produced similar tree topology, but with slightly better resolution and statistical support (Figure 3B). Acuarioidea, Physalopteroidea, Filarioidea and Habronematoidea were monophyletic, whereas Spiruroidea was paraphyletic,

Table 2 Number of microsatellites (SSR) with unit length $\geq \mathbf{2}$ by functional groups*

\begin{tabular}{cccc}
\hline Group & No. contigs & SSR (unit > =2) & Percentage \\
\hline Annotatable & 121 & 12 & $9.9 \%$ \\
Function unknown & 90 & 13 & $14.4 \%$ \\
No hits & 137 & 26 & $19.0 \%$ \\
Total & 348 & 51 & $14.7 \%$ \\
\hline
\end{tabular}

* See Additional file 2: Table S2 for a complete list of microsatellite sequences. intermixed with other families. Among them, O. petrowi was clustered with Streptopharagus and Spirocerca, which in turn formed a sister branch to the Filarioidea, albeit with low posterior probability and bootstrap proportion support (Figure 3B). At the moment, more sophisticated phylogenetic analyses were unachievable due to the lack of more sequences from closely related species, and the lack of sufficient sequence data such as the mitochondrial genomes and proteins within Spirurida, particularly among Thelazioidea. Nonetheless, our study revealed that Thelazioidea, including quail eye worm, was closely related to filarial nematodes, which implies that therapeutic strategies for filariasis such as those for L. loa might be referential in developing treatments for the Thelazoidea eye worms.

\section{Feature of internal transcribed regions and molecular detection of 0 . petrowi}

In addition to the nearly complete $18 \mathrm{~S}$ rRNA gene, we have also determined the complete sequences of the ITS1, 5.8S rRNA and ITS2 regions. Two types of ITS1 sequences have been observed (GenBank accession numbers: KF110799 for type 1 and KF110800 for type 2 ITS1), in which the type 1 sequence contained a 17nucleotide insertion at positions 1911 - 1927 (Figure 4). Additionally, there were two nucleotide differences between the two types of ITS1 sequences at positions 2112 and 2188 (in reference to KF110799). The insertion/deletion was further confirmed by PCR using primers flanking the region and subsequent sequencing of PCR amplicons.

While the 5.8S rRNA gene shared significant sequence homology to those of other nematodes available the NCBI databases (data not shown), both the ITS1 and ITS2 regions displayed high sequence diversity (i.e., no significant hits in BLASTN searches of the NCBI nucleotide databases). Because of the insertion/deletion at the ITS1 region, we initially designed two pairs of primers based on the ITS2 sequence for the potential of using nested PCR-based molecular detection of $O$. petrowi: an external primer pair QEW_2373F (5'-AAG AAT GTA ATG TTG TGG AGC-3') and QEW_2681R (5'-GTA ATC ACA TTT GAG TTG AGG-3'), and an internal primer pair QEW_2417F and QEW_2578R (as described in the Methods section) that would give $309 \mathrm{bp}$ and $162 \mathrm{bp}$ products, respectively. However, after our pilot experiments indicated that nested PCR was unnecessary, we used regular $\mathrm{qPCR}$ reactions with the QEW_2417F and QEW_2578R primers in subsequent detection of $O$. petrowi DNA from wild bobwhite fecal specimens collected in Texas in February - March, 2013. The specificity of the QEW_2417F and QEW_2578R primer pair for O. petrowi was also confirmed by its inability to produce products from DNA 


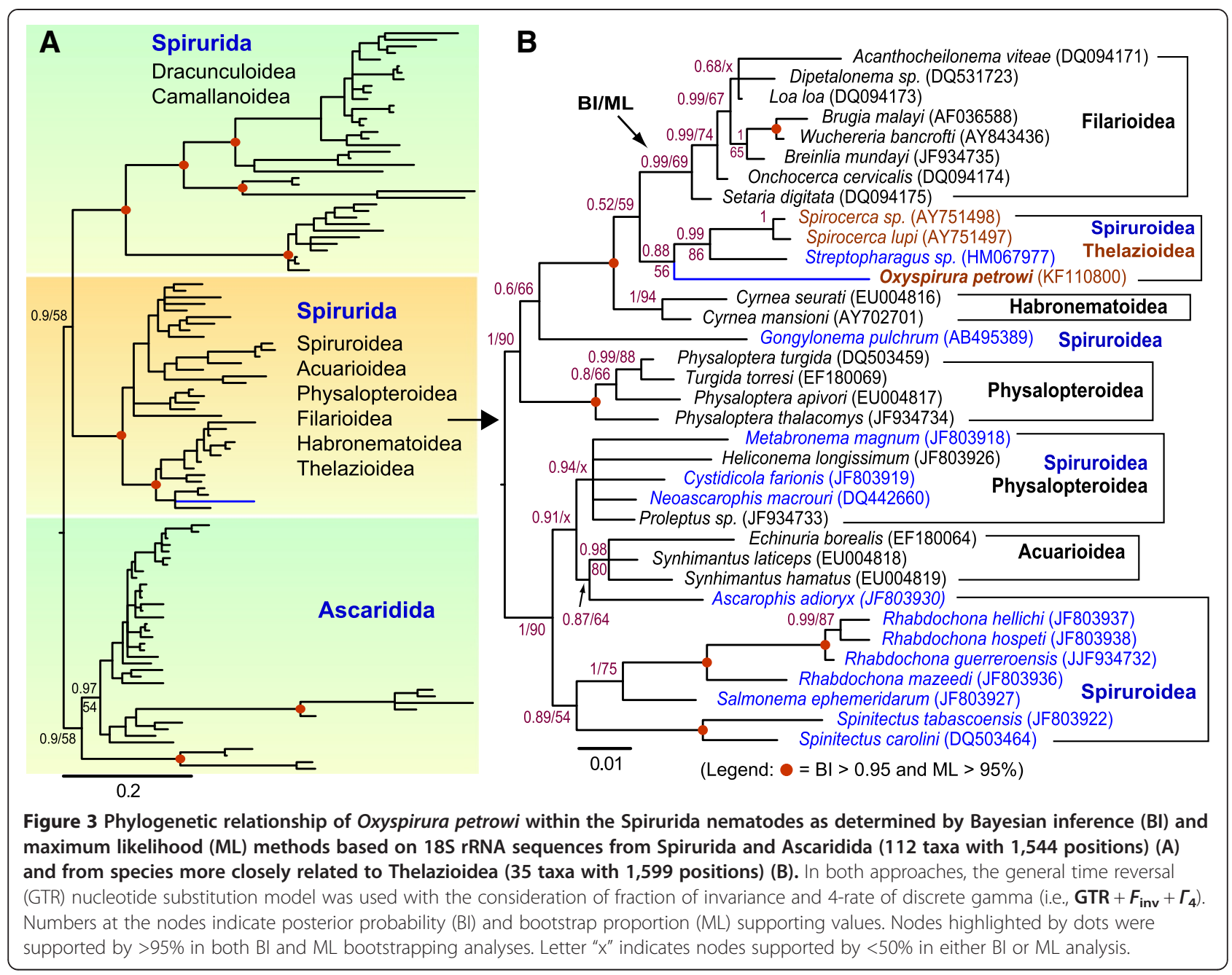

isolated from the cecal worm A. pennula that is commonly present in wild quail (Figure 5).

On the other hand, due to the lack of molecular sequences at the ITS regions from very closely related species, we could not firmly conclude that the relatively short primers were absolutely specific to O. petrowi. In fact, only six ITS1 sequences were present in the GenBank database for the superfamily Thelazioidea, including five from Thelazia species and one from $O$. conjuctivalis (accession: EF417873). However, these ITS1 sequences were highly divergent from each other and from those of $O$. petrowi (i.e., $47.5 \%$ - $48.5 \%$ identities between the O. conjuctivalis and O. petrowi and 26.1\% -
53.2\% between the five Thelazia species within the ITS1 sequences), which indicated that their ITS2 regions were likely divergent enough for speciation. To further ensure the quality of detection, selected individual or pooled PCR products were also sequenced to validate their identities. When the qPCR detection system was used, melting curve analysis was performed to confirm that PCR amplicons showed the same curves as those of positive controls.

Among the 54 fecal DNA specimens, we have detected $21(38.9 \%)$ positive samples. However, because the specimens were collected from both individual and pooled quail samples derived from 88 birds, direct calculation

ITS1-type 1: 1888 GTAAATATCTGAAAGGATGAAAAAAGCAACGCTACTTATTACCACACCTGTTTGTTTTTGTTGAAAAAAAT 1957 ITS1-type 2: 1888 GTAAATATCTGAAAGGATGAAAA-----------ACCACACCTGTTTGTTTTGTTGAAAAAAAT 1940

Figure 4 Comparison of the Oxyspirura petrowi type 1 and type 2 ITS1 sequences at the insertion/deletion site. Their sequences are available at GenBank database with accession numbers [GenBank:KF110799] and [GenBank:KF110800]. 


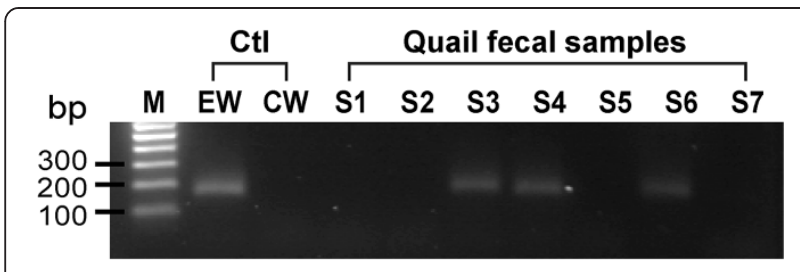

Figure 5 Agarose gel (1.5\%) electrophoresis illustrating PCRbased detection of Oxyspirura petrowi DNA from quail fecal samples. Lane M: 100-bp molecular marker; Lanes EW and CW: regular PCR using DNA isolated from adult eye worm (EW, O. petrowi) and cecal worm (CW, A. pennula) isolated from wild quail as positive and negative controls (Ct); Lanes S1 - S7: results of real-time qPCR detection from selected positive and negative stool DNA samples.

of positive rate (i.e., $21 / 54=38.9 \%$ ) was inappropriate as pooled positive specimens might contain both positive and negative samples. Therefore, we employed two additional approaches to estimate the prevalence. The first approach was to only calculate positive rate from the 39 samples collected from individual birds (non-pooled), in which 13 samples were positive, giving a 33.3\% positive rate. The second approach employed software written by Dr. Brad Biggerstaff as an Excel Add-In (PooledInfRate, version 3.0) (http://www.cdc.gov/westnile/resourcepages/ mosqSurvSoft.html), which was originally developed to determine positive rates of viral infections in pooled mosquito samples using a maximum likelihood estimation (MLE) algorithm [22]. By applying a bias-corrected MLE estimation, we obtained an infection rate of $27.7 \%$ with lower and upper limits at $18.6 \%$ and $38.7 \%$, respectively (95\% confidence interval). Collectively, we conclude that $\sim 30 \%$ (or between $28 \%$ - 33\%) of the sampled wild quail were infected by the eye worm. The actual rate might be even higher, as the fecal samples were only collected once, rather than continuously for several days, and the sensitivity of PCR detection might not be maximal due to the inhibitory substances commonly present in fecal samples as discussed below.

The detection of $O$. petrowi DNA in feces allows rapid and sensitive detection of the presence of eye worms without the need to examine individual birds. However, one needs to be aware of the presence of inhibitory substances in fecal samples and the difficulties in releasing DNA from eggs or encysted larvae. The presence of inhibitory substances could be minimized (if not completely eliminated) using the tablets included in the DNA isolation kits specifically designed for stool samples such as the QIAamp DNA Stool Mini Kit (Qiagen). Freeze/thaw cycles combined with homogenization with glass beads were necessary to break the eggs or encysted larvae to ensure the release of DNA. Furthermore, nested PCR might also be used by the addition of a primary amplification using the external primers QEW_ 2373F and QEW_2681R to not only improve the sensitivity of PCR detection, but to also further eliminate the presence of inhibitory substances in a second amplification procedure.

The life cycle of O. petrowi is not well understood, its exact intermediate host(s) as well as its migration details in quail. The presence of O. petrowi DNA in quail feces indicates that eggs laid by female adult worms in the eyes could be passed into the digestive system and released with the excrement via the naso-lacrimal ducts to the gut route, as previously observed in other eye worms such as O. mansoni [23].

This PCR detection protocol can also be easily adapted to identify intermediate host(s) and perform surveillance, which is important in developing effective strategies to control the transmission of eye worms in the field. Our phylogenetic analysis based on $18 \mathrm{~S}$ rRNA sequences indicated that $O$. petrowi clustered closely with Streptopharagus and Spirocerca (Figure 3). It is known that birds are a paratenic host for Spirocerca lupi infection in their life cycle between dogs and dung beetles [24]. Dung beetles are also the intermediate host for Streptopharagus [25]. These observations suggest that dung beetles might be worth examining as one of the potential intermediate hosts. Indeed, the detection of $O$. petrowi DNA in various insects including dung beetles is currently ongoing as part of a separate project in determining the intermediate host(s) and transmission route(s), and the data will be reported upon the completion of the survey.

\section{Conclusions}

We have performed a small-scale genome sequence survey (GSS), which not only rapidly generated a large number of molecular sequence data for the first time for O. petrowi, but also provided a snapshot of the genome for the eye worm in quail. The survey also identified a large number of microsatellite sequences that may be employed in further genotyping and population genetics studies. Our phylogenetic reconstructions based on $18 \mathrm{~S}$ rRNA sequences indicated that Spiruroidea was paraphyletic, while O. petrowi, Strepto pharagus and Spirocerca formed a sister clade to the filarial nematodes. The obtained ITS sequence data also permitted us to design specific primers for molecular detection of $O$. petrowi in fecal samples, which may also be adapted to detect this nematode in insect intermediate hosts for surveillance and developing strategies to control the transmission of eye worms from intermediate hosts to quail. We also determined that $28 \%-33 \%$ of the birds were O. petrowi positive, suggesting that eye worm was a significant parasite in at least some quail ranches in Texas. 


\section{Additional files}

Additional file 1: Table S1. List of contigs with annotations and information on top blast hits.

Additional file 2: Table S2. Oxyspirura petrowi microsatellite sequences identified by the GSS (all perfect matches) using Phobos.

\section{Competing interests}

The authors declare that they have no competing interests.

\section{Authors' contributions}

LX participated in experimental design and performed the majority of experiments on the genome survey including constructing genomic library, cloning and sequencing, the cloning and sequencing of rRNA gene and downstream region sequences, and the isolation stool DNA and PCR/qPCR detection; FG and $\mathrm{HZ}$ participated in sample preparation; $\mathrm{LL}$ participated in collection of fecal samples from wild quail; $A B$ participated in collection of adult eye worms; DR participated in fecal sample collection, writing the manuscript, and securing funding for the study; AMF participated in collection and speciation of eye worm and writing manuscript; GZ conceived the study, participated in its design, molecular and phylogenetic analysis, and writing the manuscript. All authors read and approved the final manuscript.

\section{Acknowledgements}

Major funding for this research provided by Rolling Plains Quail Research Foundation (http://www.quailresearch.org) to GZ, AMF and DR. We thank Dr. Jason M. Fritzler at the Weber State University for his critical reading of the manuscript.

\section{Author details}

${ }^{1}$ Department of Veterinary Pathobiology, College of Veterinary Medicine \& Biomedical Sciences, Texas A\&M University, College Station, Texas, USA. ${ }^{2}$ College of Life Sciences, Zhejiang University, Hangzhou, Zhejiang, China. ${ }^{3}$ Texas A\&M AgriLife Research, San Angelo, Texas, USA. ${ }^{4}$ Rolling Plains Quail Research Ranch, Rotan, Texas, USA. ${ }^{5}$ Caesar Kleberg Wildlife Research Institute, Texas A\&M University-Kingsville, Kingsville,Texas, USA.

Received: 15 June 2013 Accepted: 14 October 2013 Published: 21 October 2013

\section{References}

1. Pence DB: The genus Oxyspirura (nematoda: thelaziidae) from birds in Louisiana. Proc Helminth Soc Washington 1972, 39(1):23-28.

2. Landgrebe JN, Vasquez B, Bradley RG, Fedynich AM, Lerich SP, Kinsella JM: Helminth community of scaled quail (Callipepla squamata) from western Texas. J Parasitol 2007, 93(1):204-208.

3. Jackson AS: A handbook for bobwhite quail management in west Texas rolling plains. Texas Parks: Wildlife Department; 1969.

4. Villarreal SM: Helminth infections across the annual breeding cycle of northern bobwhites from Fisher County, Texas. Kingsville, TX: Texas A\&M UniversityKingsville; 2012.

5. Addison EM, Prestwood AK: Oxyspirura turcottei n.sp. (Nematoda: Thelaziidae) from the eastern wild turkey (Meleagris gallopavo silvestris). Can J Zool 1978, 56(5):1218-1221.

6. Ali SM: On some new species of the genus Oxyspirura from birds in Hyderabad, Andhra Pradesh, India. J Helminthol 1960, 34:221-242.

7. Ivanova E, Spiridonov S, Bain O: Ocular oxyspirurosis of primates in zoos: intermediate host, worm morphology, and probable origin of the infection in the Moscow zoo. Parasite 2007, 14(4):287-298.

8. Jairapuri DS, Siddiqi AH: A review of the genus Oxyspirura Drasche in Stossich, 1897 (Nematoda: Thelaziidae) with descriptions of fourteen new species. J Helminthol 1967, 41(4):337-363.

9. Schwabe CW: Studies on Oxyspirura mansoni, the tropical eyeworm of poultry. III. Preliminary observations on eyeworm pathogenicity. Am J Vet Res 1950, 11(40):286-290

10. Vellayan S, Jeffery J, Oothuman P, Zahedi M, Krishnasamy M, Paramaswaran S, Rohela M, Abdul-Aziz NM: Oxyspiruriasis in zoo birds. Trop Biomed 2012, 29(2):304-307.
11. Hu ZL, Bao J, Reecy JM: CateGOrizer: a Web-based program to batch analyze gene ontology classification categories. Onl J Bioinform 2008, 9(2):108-112.

12. Moriya Y, Itoh M, Okuda S, Yoshizawa AC, Kanehisa M: KAAS: an automatic genome annotation and pathway reconstruction server. Nucleic Acids Res 2007, 35:182-185.

13. Schattner P, Brooks AN, Lowe TM: The tRNAscan-SE, snoscan and snoGPS web servers for the detection of tRNAs and snoRNAs. Nucleic Acids Res 2005, 33:686-689.

14. Gardner PP, Daub J, Tate J, Moore BL, Osuch IH, Griffiths-Jones S, Finn RD, Nawrocki EP, Kolbe DL, Eddy SR, et al: Rfam: wikipedia, clans and the "decimal" release. Nucleic Acids Res 2011, 39:141-145.

15. Edgar RC: MUSCLE: a multiple sequence alignment method with reduced time and space complexity. BMC Bioinforma 2004, 5:113.

16. Edgar RC: MUSCLE: multiple sequence alignment with high accuracy and high throughput. Nucleic Acids Res 2004, 32(5):1792-1797.

17. Jobb G, von Haeseler A, Strimmer K: TREEFINDER: a powerful graphical analysis environment for molecular phylogenetics. BMC Evol Biol 2004, 4:18.

18. Ronquist F, Teslenko M, van der Mark P, Ayres DL, Darling A, Hohna S, Larget B, Liu L, Suchard MA, Huelsenbeck JP: MrBayes 3.2: efficient Bayesian phylogenetic inference and model choice across a large model space. Syst Biol 2012, 61(3):539-542.

19. Smythe $A B$, Sanderson MJ, Nadler SA: Nematode small subunit phylogeny correlates with alignment parameters. Syst Biol 2006, 55(6):972-992.

20. Meldal BH, Debenham NJ, De Ley P, De Ley IT, Vanfleteren JR, Vierstraete AR, Bert W, Borgonie G, Moens T, Tyler PA, et al: An improved molecular phylogeny of the Nematoda with special emphasis on marine taxa. Mol Phylogenet Evol 2007, 42(3):622-636.

21. Gelman A, Rubin DB: Inference from iterative simulation using multiple sequences. Stat Sci 1992, 7(4):457-472.

22. Hepworth G: Confidence intervals for proportions estimated by group testing with groups of unequal size. J Agr Biol Envir St 2005, 10(4):478-497.

23. Schwabe CW: Studies on Oxyspirura mansoni, the tropical eyeworm of poultry, II. Life history. Pacific Sci 1951, 5(1):18-35.

24. Oryan A, Sadjjadi SM, Mehrabani D, Kargar M: Spirocercosis and its complications in stray dogs in Shiraz, southern Iran. Vet Med 2008 53(11):617-624.

25. Boze BGV, Hernandez AD, Huffman MA, Moore J: Parasites and dung beetles as ecosystem engineers in a forest ecosystem. J Insect Behav 2012, 25(4):352-361.

doi:10.1186/1471-2180-13-233

Cite this article as: Xiang et al:: Gene discovery, evolutionary affinity and molecular detection of Oxyspirura petrowi, an eye worm parasite of game birds. BMC Microbiology 2013 13:233.

\section{Submit your next manuscript to BioMed Central and take full advantage of:}

- Convenient online submission

- Thorough peer review

- No space constraints or color figure charges

- Immediate publication on acceptance

- Inclusion in PubMed, CAS, Scopus and Google Scholar

- Research which is freely available for redistribution 